\title{
Evidências da Polarização Epidemiológica no Nordeste Brasileiro: Análise Espacial Pelas Técnicas de Regionalização e de Aglomerados de Áreas
}

\section{Evidence of Epidemiological Polarization in Northeastern Brazil: Spatial Analysis Using Regionalization Techniques and Area Clusters}

\author{
Sávio Marcelino Gomes ; ; Ana Mayara Gomes de Souza ; Talita Araujo de Souza*a; Isabelle Ribeiro Barbosa ${ }^{a}$
}

${ }^{a}$ Universidade Federal do Rio Grande do Norte, Programa de Pós-Graduação Stricto Sensu em Saúde Coletiva. RN, Brasil.

E-mail: talitaaraujo23@hotmail.com

\begin{abstract}
Resumo
Os padrões epidemiológicos da mortalidade humana sofrem alterações ao longo dos anos, sendo necessário monitorar indicadores de mortalidade para avaliar a transição epidemiológica, considerando diferentes dimensões do território. O objetivo desse estudo foi identificar padrões espaciais de mortalidade no nordeste brasileiro. Estudo ecológico com 1794 municípios nordestinos, onde foram utilizados os óbitos registrados no Sistema de Informação sobre Mortalidade. Foram analisadas as proporções de mortalidade por capítulos CID-10 pelos métodos aglomerativo hierárquico e de Skater no software Geoda 1.14.0.24. Foram registrados 1.673.016 de óbitos ocorridos em residentes da região nordeste do Brasil. Por ambas as técnicas, foi identificado um cluster de elevada mortalidade por câncer e por doenças do aparelho circulatório e respiratório nos estados do Rio Grande do Norte, Paraíba e Pernambuco; um cluster de elevada mortalidade por doenças infecciosas, morte materna e problemas relacionados ao período perinatal no estado do Maranhão e um cluster de elevada mortalidade por Transtornos mentais e comportamentais em municípios do estado de Sergipe, Piauí e Ceará. Pela técnica de Skater foi identificado um cluster de elevada mortalidade por doenças endócrinas nutricionais e metabólicas, doenças do aparelho digestivo, doenças do sistema osteomuscular e do tecido conjuntivo, doenças do aparelho geniturinário em uma grande área que abrange os municípios dos estados do Rio Grande do Norte, Paraíba, Pernambuco e Alagoas. Foi possível identificar padrões de mortalidade no Nordeste, e as técnicas aplicadas conseguiram identificaram padrões semelhantes.

Palavras-chave: Mortalidade. Classificação Internacional de Doenças. Análise Espacial. Regionalização. Análise de Agrupamentos.
\end{abstract}

\begin{abstract}
Epidemiological and human mortality patterns undergo changes over the years, so it is necessary to monitor mortality indicators to assess the epidemiological transition, considering different dimensions of the territory. This is study objective identifying spatial patterns of mortality in Brazilian Nordeste region. Ecological study ranging 1794 cities and towns in that region, where we used the records of death from the Sistema de Informação sobre Mortalidade (Mortality Register Information System, in a free translation from Portuguese). We analyzed the proportions of mortality by ICD-10 chapters according to hierarchic agglomerative and Skater methods in Geoda 1.14.0.24 software. 1.673.016 deaths were recorded among people who live in Brazilian Nordeste region. According to both techniques we used, we identified a cluster of high mortality rate caused by cancer and circulatory and respiratory diseases in Rio Grande do Norte, Paraiba and Pernambuco states; another cluster of high mortality rate because of infectious diseases, maternal death and problems related to perinatal period in Maranhão state; besides a cluster of high mortality rates because of behavioral and mental disorders in cities and towns of Sergipe, Piaui and Ceará states. According to Skater technique, we identified a cluster of high mortality rate caused by nutritional endocrine and metabolic diseases, digestive system diseases and musculoskeletal system and connective tissue diseases, besides genitourinary system diseases in a large area evolving cities and towns of Rio Grande do Norte, Paraiba, Pernambuco and Alagoas states. We could identify mortality patterns in Brazilian Nordeste region, and the techniques we applied for it identified the same patterns.
\end{abstract}

Keywords: Mortality. International Disease Code. Spatial Analysis. Regionalization. Group Analysis.

\section{Introdução}

Os padrões epidemiológicos de mortalidade nas populações humanas vêm passando por transformações, deixando de se concentrar em faixas etárias mais jovens e em grande parte por doenças infecciosas e deficiências nutricionais, para padrões de mortalidade estáveis, concentrados em idades mais antigas e causadas principalmente por doenças e agravos não transmissíveis. A associação desses padrões com as transformações industriais, demográficas, sanitárias, tecnológicas, ambientais e comportamentais resultou na teoria - ou modelo - de transição epidemiológica, cunhada por Abdel Omran no início dos anos 70 (KABUDULA et al., 2017; MENDOZA; MIRANDA, 2017).

Estudos realizados com diversos países de todos os continentes demonstraram que houve uma diminuição de cerca de $40 \%$ das taxas de mortalidade padronizadas por idade, entre 1990 e 2013, para as doenças infecciosas, mortalidade materna, infantil e por causas nutricionais, em contrapartida, a proporção de mortes por doenças e agravos não transmissíveis aumentou $42 \%$ no mesmo período. As mudanças nos padrões refletem os impactos das intervenções nos sistemas de saúde e das estratégias governamentais empregadas para a redução de 
fatores de risco na população (ALI et al., 2015).

No entanto, as mudanças nos padrões de mortalidade não ocorreram de maneira uniforme entre regiões e países. As situações epidemiológicas de diferentes regiões em um mesmo país tornam-se contrastantes é o que caracteriza a polarização epidemiológica (SCHRAMM et al., 2004). Entre 1980 e 2012, nos países desenvolvidos, a mortalidade por doenças e agravos não transmissíveis, em geral, passou a diminuir com o tempo, com exceção das mortes por diabetes e alguns tipos de neoplasias, que se mantinham relativamente estáveis. Nos países subdesenvolvidos ou em desenvolvimento, por sua vez, as mortes por doenças cardiovasculares, por diabetes e neoplasias apresentavam tendência de crescimento, com persistência da mortalidade por causas relacionadas à pobreza (ALI et al., 2015; KABUDULA et al., 2017).

Os países da América Latina podem ser divididos em dois grupos: aqueles que alcançam um maior número médio de anos vividos na idade adulta, e o segundo com países que possuem o menor número médio. Embora o padrão de mortalidade por causa seja semelhante entre todos os países, os níveis entre elas se diferenciam. No primeiro grupo, a mortalidade por doenças e agravos não transmissíveis começou a reduzir, já nos países do segundo grupo, continuou a subir entre 1950 e 2000 (CALAZANS; QUEIROZ, 2020).

No Brasil, no período de 1990 a 2010, a expectativa de vida aumentou de 65,4 para 70,5 anos em homens e de 73,1 para 77,7 anos em mulheres. Nesse período, as principais causas de mortalidade foram derivadas de doenças cardiovasculares, em detrimento de aumento de mortes por diabetes e doença renal e redução da mortalidade por diarreia (MARINHO; PASSOS; FRANÇA, 2016). No entanto, há indícios de polarização epidemiológica entre as regiões brasileiras, definida pela coexistência de elevada prevalência de doenças infecciosas em determinadas regiões, em especial nas regiões Norte e Nordeste, fruto de um desenvolvimento historicamente desigual (ARAÚJO, 2012).

O Brasil está no ranking entre os países com as maiores desigualdades sociais e econômicas do mundo, apesar do substancial desenvolvimento vivido pelo país nas últimas décadas. O Índice de Desenvolvimento Humano nas regiões Sudeste, Sul e Centro-Oeste do país varia em cerca de 0,75 0,76 , enquanto nas regiões Norte e Nordeste variam entre 0,66-0,67 (RODRIGUES et al., 2016). A região Nordeste revela um elevado grau de desigualdade, expressa tanto em uma dimensão econômica quanto nas relações de acesso aos serviços públicos, educação e habitação, resultando em níveis distintos de saúde em relação às demais regiões do país (VIACAVA, 2019).

Nesse contexto, é pertinente a utilização dos Sistemas de Informações Geográficas (SIG), que são ferramentas computacionais do campo do geoprocessamento, para a montagem de um painel sócio-territorial ampliado para diagnosticar o perfil do processo de transição epidemiológica.
Os estudos de distribuição e análise espacial têm sido ferramentas úteis que podem contribuir com as politicas públicas, e mais diretamente à vigilância em saúde. As análises espaciais ampliam o campo da vigilância epidemiológica, no monitoramento e colaboração para o planejamento de ações em áreas de riscos e intervenções de forma articulada com outros setores, já que permite conhecer a magnitude dos fenômenos bem como identificar grupos mais vulneráveis a situações de risco.

Há uma necessidade de que o acompanhamento dos indicadores de mortalidade para avaliação da transição epidemiológica considere diferentes dimensões do território, a exemplo dos níveis estadual e municipal, tendo em vista as desigualdades existentes em determinados contextos. $\mathrm{O}$ estudo propõe, portanto, identificar os padrões espaciais de mortalidade no nordeste brasileiro, considerando suas dimensões municipais.

\section{Material e Métodos}

Trata-se de um estudo ecológico que utilizou como base geográfica os 1794 municípios do nordeste do Brasil. Para compreender a organização dos conglomerados de mortalidade por município, foram utilizados os óbitos registrados no período de 2013-2017. O número de óbitos foi obtido do Sistema de Informação sobre Mortalidade (SIM), e essas informações foram extraídas do sítio eletrônico do Departamento de Informática do SUS (datasus.saude.gov.br).

As variáveis epidemiológicas consideradas foram traduzidas por indicadores de saúde calculados por meio de proporções. Foi analisada a mortalidade proporcional por capítulo CID-10, expressa pelo quociente entre o número de óbitos ocorridos por capítulo e o total de óbitos registrados, multiplicado por 100. Optou-se por utilizar o período de 5 anos para o cálculo dos indicadores para evitar as flutuações aleatórias na ocorrência dos eventos. O software utilizado para a tabulação dos dados foi o Tabwin 3.2.

A mortalidade proporcional por Capítulo CID-10 foi analisada nos seguintes subconjuntos: (Grupo 1): Capítulo II - Neoplasias; Capítulo IX- doenças do aparelho circulatório; Capítulo X - Doenças do aparelho respiratório; Capítulo XX - Causas externas, (Grupo 2): Capítulo I- Algumas doenças infecciosas e parasitárias; Capítulo XV- Gravidez, parto e puerpério; Capítulo XVI- algumas afecções originadas do período perinatal; (Grupo 3): Capítulo III- Doenças do sangue, órgãos hematopoiéticos e transtornos imunitários; Capítulo IV - doenças endócrinas nutricionais e metabólicas; Capítulo XIDoenças do aparelho digestivo; Capítulo XII - Doenças da pele e tecido subcutâneo; Capítulo XIII- Doenças do sistema osteomuscular e do tecido conjuntivo; Capítulo XIV- Doenças do aparelho geniturinário. (Grupo 4): Capítulo V- Transtornos mentais e comportamentais; Capítulo VI- Doenças do Sistema nervoso.

Foi realizada uma análise descritiva para resumir o 
conjunto de dados, utilizando média, mediana, desvio padrão e os percentis 25 e 75 . Inicialmente, todas as variáveis foram padronizadas pelo escore $Z$ em razão do impacto que diferentes escalas podem acarretar na função de dissimilaridade e na soma de quadrados dentro dos conglomerados. Um conjunto de variáveis padronizadas deve ter média zero e desvio-padrão igual a uma unidade.

Para a análise da regionalização da mortalidade proporcional no nordeste do Brasil foi aplicada a técnica de Skater. O Skater é um método de regionalização de áreas que permite agrupar áreas menores (municípios, bairros) em áreas maiores, que, além de serem contíguas, têm também semelhança segundo os atributos associados. Constitui uma análise de conglomerados que leva em conta a localização espacial dos objetos (VASCONCELOS et al, 2012). A regionalização de áreas pelo método Skater inicia com a construção do grafo, que consiste num diagrama composto de pontos (unidades espaciais com os respectivos dados), a partir dos quais os vizinhos são ligados entre si por linhas que representa as unidades espaciais que estão inter-relacionadas. No algoritmo Skater, a montagem desse grafo é feita por uma matriz de proximidade. Atribui-se valor às arestas de acordo com a dissimilaridade (distância entre os atributos ou valores correspondentes). A partir deste grafo a Árvore Geradora Mínima é montada e o problema da regionalização torna-se equivalente ao particionamento do grafo, de modo a conseguir a melhor similaridade entre as regiões (ANDRADE, SOUZA; GOMES, 2015; INPE, 2006).

Visando a análise da interação entre as os municípios nordestinos, conduziu-se uma análise de agrupamentos hierárquico espacial para determinar o nível de proximidade entre os mesmos. A análise de cluster ou análise de aglomerados é uma técnica de análise multivariada que permite reunir objetos e/ou variáveis de acordo com suas características comuns. O objetivo desta técnica é reduzir o número de objetos, que estão dispostas em uma matriz de observação, agrupando-os em clusters, ou seja, grupos criados para reunir objetos de acordo com suas similaridades, através de critérios de similaridade previamente determinados
(CORRAR; PAULO; DIAS FILHO, 2007). A partir da padronização das variáveis em escore $\mathrm{Z}$, utilizou-se o método hierárquico aglomerativo de Ward. O procedimento básico consiste em computar uma matriz de distância ou similaridade entre os municípios, a partir da qual se inicia um processo de sucessivas fusões destes, com base na proximidade ou similaridade entre eles. Como medida de semelhança entre as observações utilizou-se o quadrado da distância euclidiana e os resultados são apresentados no dendrograma, diagrama de árvore que exibe os grupos formados por agrupamento de observações em cada passo e em seus níveis de similaridade (RIBEIRO et al., 2014).

Foi utilizada a formação de 5 clusters como base em ambas as técnicas. Para análise dos agrupamentos formados, são apresentadas as somas dos quadrados dentro de cada cluster e entre os clusters, com o intuito de mostrar a diferença entre os mesmos. Para a produção dos mapas temáticos, a base cartográfica foi obtida no site do Instituto Brasileiro de Geografia e Estatística (https://ibge.gov.br/), e para realização da técnica de Skater e de Aglomerados foi utilizado o software GeoDa 1.14.0.24 de agosto de 2019. Nesta pesquisa foram utilizados dados secundários extraídos de sites oficiais abertos à consulta pública, Dessa forma, não houve necessidade de apreciação do Comitê de Ética e Pesquisa (CEP) como preconiza a Resolução ${ }^{\circ}$ 510/2018 do Conselho Nacional de Saúde (CNS).

\section{Resultados e Discussão}

No período de 2013 a 2017, foram registrados 1.673.016 de óbitos ocorridos em residentes da região nordeste do Brasil. A análise descritiva da mortalidade proporcional para os municípios do nordeste mostra que os Capítulos IX - doenças do aparelho circulatório (30\%), Capítulo XX- Causas externas (13,5\%) e Capítulo II- Neoplasias (12,3\%) detém 55\% dos óbitos ocorridos no período de 2013 a 2017 (Quadro 1). Destaca-se que, nesse período, não foram registrados óbitos para os capítulos XIX- Lesões, envenenamento e alguma outra consequência de causas externas, XXII-Códigos para propósitos especiais e XXI- Contatos com serviços de saúde.

Quadro 1 - Análise descritiva da mortalidade proporcional por capítulo CID-10 no período 2013-2017 para os municípios do nordeste do Brasil

\begin{tabular}{|l|c|c|c|c|c|c|}
\hline Capítulos CID-10 & Mínimo & Máximo & Média & Desvio-padrão & Percentil 25 & Percentil 75 \\
\hline Capítulo I & 0,000 & 13,79 & 3,83 & 1,53 & 2,83 & 4,64 \\
\hline Capítulo II & 0,000 & 41,66 & 12,30 & 3,31 & 10,07 & 14,34 \\
\hline Capítulo III & 0,000 & 9,16 & 0,631 & 0,54 & 0,29 & 0,86 \\
\hline Capítulo IV & 0,000 & 21,21 & 7,99 & 2,85 & 6,12 & 9,72 \\
\hline Capítulo V & 0,0000 & 6,28 & 1,13 & 0,84 & 0,55 & 1,55 \\
\hline Capítulo IV & 0,000 & 9,41 & 1,68 & 1,02 & 1,02 & 2,17 \\
\hline Capítulo VII & 0,000 & 0,91 & 0,00 & 0,02 & 0,00 & 0,00 \\
\hline Capítulo VIII & 0,000 & 1,44 & 0,01 & 0,05 & 0,00 & 0,00 \\
\hline Capítulo IX & 1,081 & 60,00 & 30,05 & 6,42 & 25,61 & 34,17 \\
\hline Capítulo X & 0,000 & 24,16 & 9,14 & 3,17 & 6,86 & 11,07 \\
\hline Capítulo XI & 0,000 & 13,41 & 4,75 & 1,51 & 3,82 & 5,58 \\
\hline
\end{tabular}


Continuação...

\begin{tabular}{|l|c|c|c|c|c|c|}
\hline Capítulos CID-10 & Mínimo & Máximo & Média & Desvio-padrão & Percentil 25 & Percentil 75 \\
\hline Capítulo XII & 0,000 & 6,60 & 0,36 & 0,42 & 0,00 & 0,56 \\
\hline Capítulo XIII & 0,000 & 4,47 & 0,33 & 0,42 & 0,00 & 0,50 \\
\hline Capítulo XIV & 0,000 & 13,51 & 1,97 & 1,03 & 1,34 & 2,49 \\
\hline Capítulo XV & 0,000 & 2,94 & 0,21 & 0,31 & 0,00 & 0,31 \\
\hline Capítulo XVI & 0,000 & 11,11 & 2,29 & 1,33 & 1,46 & 2,85 \\
\hline Capítulo XVII & 0,000 & 9,09 & 0,90 & 0,65 & 0,50 & 1,19 \\
\hline Capítulo XVIII & 0,000 & 52,92 & 8,85 & 8,11 & 3,37 & 11,41 \\
\hline Capítulo XX & 0,000 & 64,08 & 13,48 & 4,66 & 10,57 & 15,62 \\
\hline
\end{tabular}

Fonte: Sistema de Informação sobre Mortalidade (2020)

$\mathrm{Na}$ análise dos clusters formados pela técnica de Skater no Grupo 1 (Doenças e Agravos Crônicos Não TransmissíveisDANT), observa-se a formação de um cluster de elevadas taxas de mortalidade por causas externas entre os estados de Sergipe e Alagoas. No estado do Ceará, formou um cluster de elevada mortalidade por câncer e por doenças do aparelho respiratório; Entre os estados do Maranhão e Piauí formou um cluster de elevada mortalidade por doenças do aparelho circulatório; O cluster 1, composto pelos estados do Rio Grande do Norte, Paraíba e Pernambuco, é de elevada mortalidade por câncer e por doenças do aparelho circulatório e respiratório. E em toda extensão do estado da Bahia, formou um cluster de municípios de baixa mortalidades por doenças e agravos crônicos não transmissíveis. Para o Grupo 2 (Doenças Infecciosas e Morte Materna), observa-se a formação de um cluster de elevada mortalidade por doenças infecciosas, morte materna e problemas relacionados ao período perinatal entre os municípios dos estados do Maranhão e Piauí. Para o grupo 3 (Doenças em Tecidos, Órgãos e Sistemas), houve formação de um cluster de elevada mortalidade por doenças endócrinas nutricionais e metabólicas, doenças do aparelho digestivo, doenças do sistema osteomuscular e do tecido conjuntivo, doenças do aparelho geniturinário em uma grande área que abrange os municípios dos estados do Rio Grande do Norte, Paraíba, Pernambuco e Alagoas. E para o Grupo 4 (Transtornos Mentais e Sistema Nervoso), destaca-se o cluster formado por municípios no estado de Sergipe com elevada mortalidade por transtornos mentais. Essas informações estão descritas na Figura 1 e Quadro 2.

Figura 1 - Técnica de Skater com 5 clusters para análise dos padrões de mortalidade proporcional por capítulos CID-10 nos municípios do Nordeste do Brasil no período 2013-2017. Figura A: grupo 1; Figura B: grupo 2; Figura C: grupo 3; Figura D: grupo 4
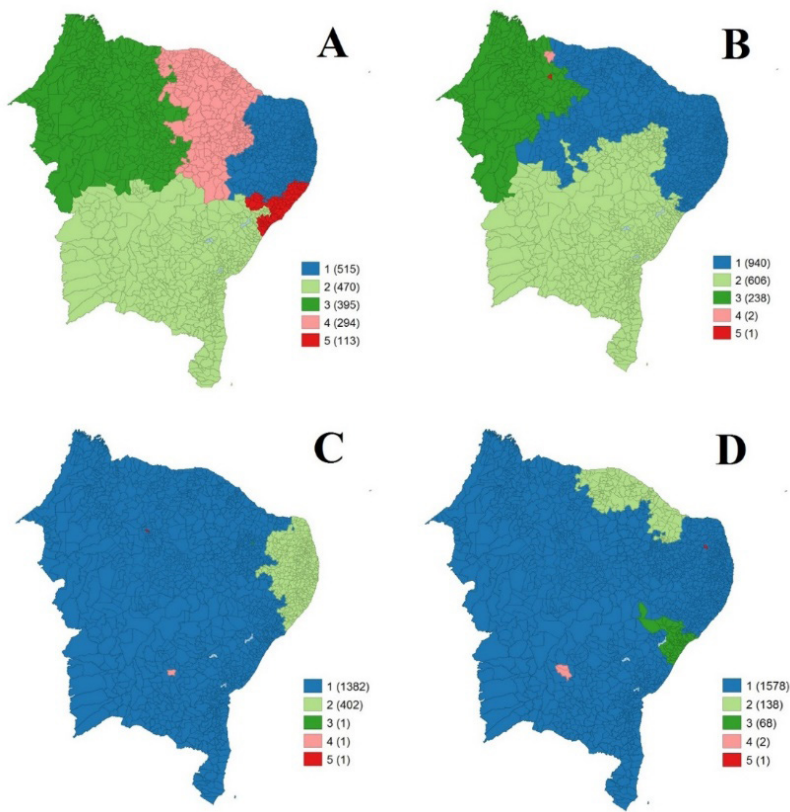

Fonte: Adaptado de Sistema de Informação sobre Mortalidade

Quadro 2 - Parâmetros dos agrupamentos e interpretação do perfil dos clusters obtidos pela técnica de Skater

\begin{tabular}{|c|c|c|c|c|c|}
\hline & Cluster 1 & Cluster 2 & Cluster 3 & Cluster 4 & Cluster 5 \\
\hline \multicolumn{6}{|c|}{ Grupo 1 - Doenças e Agravos Crônicos Não Transmissíveis } \\
\hline Capítulo II & 13,13 & 11,44 & 11,19 & 14,64 & 10,09 \\
\hline Capítulo IX & 30,87 & 25,66 & 35,31 & 29,78 & 28,44 \\
\hline Capítulo X & 10,38 & 7,49 & 7,57 & 11,87 & 8,99 \\
\hline Capítulo XX & 12,24 & 13,42 & 13,60 & 13,37 & 17,59 \\
\hline $\begin{array}{c}\text { Soma dos } \\
\text { quadrados }\end{array}$ & 1660,58 & 1395,69 & 1296,52 & 986,928 & 216,929 \\
\hline Perfil & $\begin{array}{l}\text { Elevada mortalidade } \\
\text { por câncer e } \\
\text { doenças do aparelho } \\
\text { circulatório e } \\
\text { respiratório }\end{array}$ & $\begin{array}{l}\text { Baixas taxas de } \\
\text { mortalidade }\end{array}$ & $\begin{array}{l}\text { Elevada mortalidade } \\
\text { por doenças do } \\
\text { aparelho circulatório }\end{array}$ & $\begin{array}{l}\text { Elevada mortalidade } \\
\text { por câncer e } \\
\text { doenças do aparelho } \\
\text { respiratório }\end{array}$ & $\begin{array}{l}\text { Elevada mortalidade } \\
\text { por causas externas }\end{array}$ \\
\hline \multicolumn{6}{|c|}{ Grupo 2 - Doenças Infecciosas e Morte Materna } \\
\hline Capítulo I & 3,7 & 3,95 & 3,97 & 6,66 & 3,49 \\
\hline Capítulo XV & 0,17 & 0,19 & 0,38 & 2,59 & 2,79 \\
\hline Capítulo XVI & 1,90 & 2,45 & 3,39 & 8,62 & 7,69 \\
\hline $\begin{array}{l}\text { Soma dos } \\
\text { quadrados }\end{array}$ & 2179,57 & 1566,37 & 1001,15 & 2,45 & 0 \\
\hline
\end{tabular}


Continuação..

\begin{tabular}{|c|c|c|c|c|c|}
\hline & Cluster 1 & Cluster 2 & Cluster 3 & Cluster 4 & Cluster 5 \\
\hline Perfil & $\begin{array}{l}\text { Baixas taxas de } \\
\text { mortalidade }\end{array}$ & $\begin{array}{c}\text { Elevada mortalidade } \\
\text { por doenças } \\
\text { infecciosas } \\
\text { e problemas } \\
\text { relacionados ao } \\
\text { período perinatal }\end{array}$ & $\begin{array}{l}\text { Elevada mortalidade } \\
\text { por doenças } \\
\text { infecciosas, morte } \\
\text { materna e problemas } \\
\text { relacionados ao } \\
\text { período perinatal }\end{array}$ & $\begin{array}{l}\text { Elevada mortalidade } \\
\text { por doenças } \\
\text { infecciosas, morte } \\
\text { materna e problemas } \\
\text { relacionados ao } \\
\text { período perinatal }\end{array}$ & $\begin{array}{l}\text { Elevada mortalidade } \\
\text { materna e por } \\
\text { problemas } \\
\text { relacionados ao } \\
\text { período perinatal }\end{array}$ \\
\hline \multicolumn{6}{|c|}{ Grupo 3 - Doenças em Tecidos, Órgãos e Sistemas } \\
\hline Capítulo III & 0,65 & 0,53 & 0 & 9,16 & 2,70 \\
\hline Capítulo IV & 7,61 & 9,43 & 4,71 & 8,05 & 10,81 \\
\hline Capítulo XI & 4,56 & 5,41 & 1,88 & 6,66 & 5,40 \\
\hline Capítulo XII & 0,34 & 0,40 & 6,60 & 0 & 2,70 \\
\hline Capítulo XIII & 0,31 & 0,44 & 0 & 0,13 & 0 \\
\hline Capítulo XIV & 1,84 & 2,40 & 0,94 & 3,47 & 13,51 \\
\hline $\begin{array}{c}\text { Soma dos } \\
\text { quadrados }\end{array}$ & 7196,91 & 2407,9 & 0 & 0 & 0 \\
\hline Perfil & $\begin{array}{l}\text { Baixas taxas de } \\
\text { mortalidade }\end{array}$ & $\begin{array}{l}\text { Elevadas taxas de } \\
\text { mortalidade por } \\
\text { Doenças do sistema } \\
\text { osteomuscular }\end{array}$ & $\begin{array}{l}\text { Elevada mortalidade } \\
\text { por Doenças da pele e } \\
\text { tecido subcutâneo }\end{array}$ & $\begin{array}{l}\text { Elevada mortalidade } \\
\text { por Doenças do } \\
\text { sangue, orgãos } \\
\text { hematopoieticos } \\
\text { e transtornos } \\
\text { imunitarios. }\end{array}$ & $\begin{array}{l}\text { Elevada mortalidade } \\
\text { por doenças } \\
\text { endócrinas e do } \\
\text { aparelho geniturinário }\end{array}$ \\
\hline \multicolumn{6}{|c|}{ Grupo 4 - Transtornos Mentais e Sistema Nervoso } \\
\hline Capítulo V & 1,03 & 1,64 & 2,39 & 1,19 & 0 \\
\hline Capítulo VI & 1,62 & 2,21 & 1,86 & 7,33 & 9,41 \\
\hline $\begin{array}{l}\text { Soma dos } \\
\text { quadrados }\end{array}$ & 2701,65 & 335,43 & 140,68 & 4,77 & 0 \\
\hline Perfil & Baixa mortalidade & $\begin{array}{l}\text { Valores médios de } \\
\text { mortalidade }\end{array}$ & $\begin{array}{l}\text { Elevada mortalidade } \\
\text { por transtornos } \\
\text { mentais }\end{array}$ & $\begin{array}{l}\text { Elevada mortalidade } \\
\text { por Doenças do } \\
\text { Sistema nervoso }\end{array}$ & $\begin{array}{l}\text { Elevada mortalidade } \\
\text { por doenças do } \\
\text { Sistema nervoso e } \\
\text { baixa mortalidade por } \\
\text { transtornos mentais }\end{array}$ \\
\hline
\end{tabular}

Fonte: Sistema de Informação sobre Mortalidade (2020)

Já a Figura 2 e o Quadro 3 descrevem os resultados da Análise de Agrupamentos. Para o Grupo 1, houve a formação de um cluster de elevada mortalidade por câncer e problemas respiratórios em grande extensão dos estados do Ceará, Pernambuco, Paraíba e Rio Grande do Norte; outro cluster de baixas proporções de mortalidade no interior da Bahia e um cluster de elevadas taxas de mortalidade por causas externas em toda a faixa litorânea do nordeste, bem como em áreas no interior dos estados do Maranhão e Bahia. Para o Grupo 2 , houve a formação de clusters de municípios com elevada mortalidade por afecções originadas do período perinatal no estado do Maranhão e Bahia e um cluster de baixas mortalidade materna e por afecções originadas do período perinatal entre os estado do Ceará, Rio Grande do Norte, Paraíba e Pernambuco. Para o Grupo 3, destaca-se a formação do cluster de elevada mortalidade por doenças endócrinas nutricionais e metabólicas e Doenças do aparelho digestivo e outro de elevada mortalidade por Doenças da pele e tecido subcutâneo, e Doenças do aparelho geniturinário, ambos localizados mais predominantemente nos municípios da faixa litorânea que se estende desde o estado do Rio Grande do Norte até o recôncavo baiano. E para o Grupo 4, destaca-se a formação de um cluster de elevada mortalidade por Doenças do Sistema nervoso em municípios do interior do estado do Ceará, Paraíba e Pernambuco e um cluster de elevada mortalidade por Transtornos mentais e comportamentais em municípios do estado de Sergipe, Piauí e Ceará.
Figura 2 - Técnica de aglomerados hierárquicos com 5 clusters para análise dos padrões de mortalidade proporcional por capítulos CID-10 nos municípios do Nordeste do Brasil no período 2013-2017. Figura A: grupo 1; Figura B: grupo 2; Figura C: grupo 3; Figura D: grupo 4

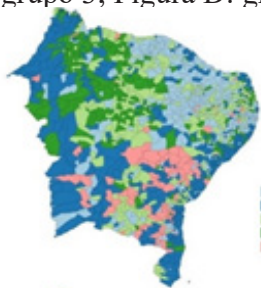

$\mathbf{A}$
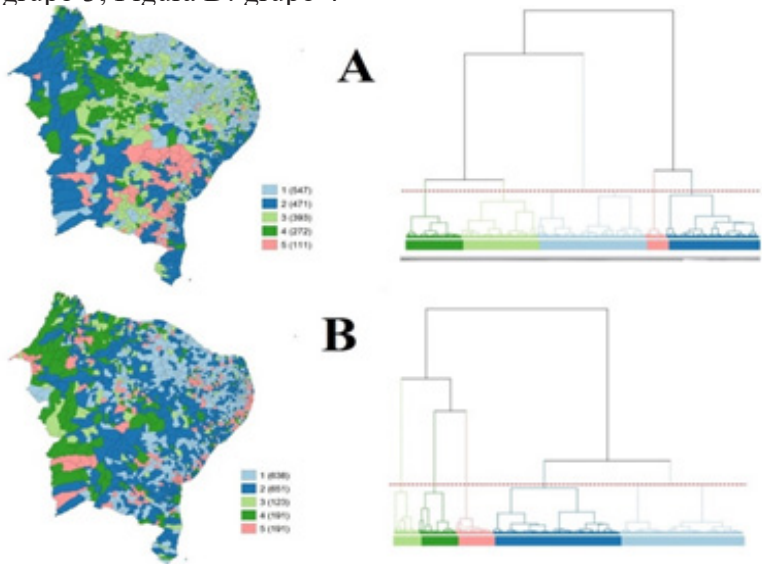

B
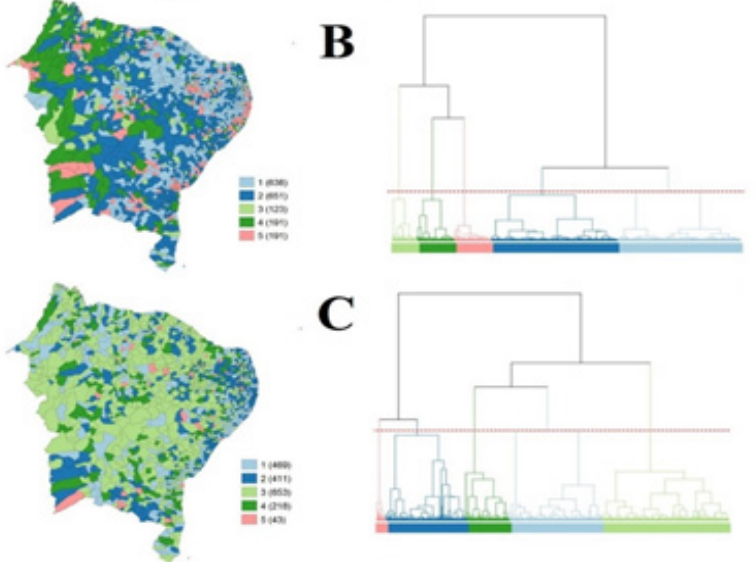

C
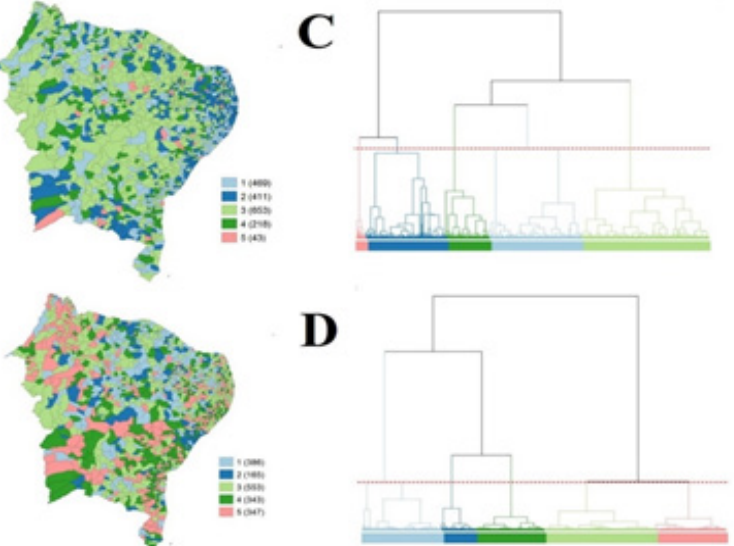

D

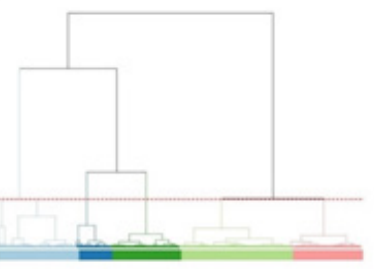

Fonte: Adaptado de Sistema de Informação sobre Mortalidade (2020). 
Quadro 3 - Parâmetros e interpretação do perfil dos clusters obtidos pela técnica de Aglomerados.

\begin{tabular}{|c|c|c|c|c|c|}
\hline & Cluster 1 & Cluster 2 & Cluster 3 & Cluster 4 & CLUSTER 5 \\
\hline \multicolumn{6}{|c|}{ Grupo 1 - Doenças e Agravos Crônicos Não Transmissíveis } \\
\hline Capítulo II & 13,84 & 10,61 & 14,21 & 10,75 & 9,33 \\
\hline Capítulo IX & 28,22 & 27,11 & 33,75 & 37,89 & 20,48 \\
\hline Capítulo X & 12,47 & 7,64 & 8,46 & 7,07 & 6,76 \\
\hline Capítulo XX & 12,72 & 17,60 & 10,42 & 12,86 & 10,33 \\
\hline $\begin{array}{l}\text { Soma dos } \\
\text { quadrados }\end{array}$ & 1424.57 & 1074.09 & 905.397 & 472.698 & 142.374 \\
\hline Perfil & $\begin{array}{c}\text { Elevada mortalidade } \\
\text { por câncer } \\
\text { e problemas } \\
\text { respiratórios } \\
\end{array}$ & $\begin{array}{l}\text { Elevada mortalidade } \\
\text { por causas externas }\end{array}$ & $\begin{array}{l}\text { Elevada mortalidade } \\
\text { por neoplasias, } \\
\text { elevada mortalidade } \\
\text { por circulatório }\end{array}$ & $\begin{array}{c}\text { Elevada mortalidade } \\
\text { circulatório }\end{array}$ & Baixa mortalidade \\
\hline \multicolumn{6}{|c|}{ Grupo 2 - Doenças Infecciosas e Morte Materna } \\
\hline Capítulo I & 3,52 & \begin{tabular}{|l|}
3,11 \\
\end{tabular} & \begin{tabular}{|l|}
3,92 \\
\end{tabular} & 4,68 & 6,38 \\
\hline Capítulo XV & 0,008 & 0,18 & 1,02 & 0,31 & 0,14 \\
\hline Capítulo XVI & 1,37 & 2,60 & 2,35 & 4,69 & 1,89 \\
\hline $\begin{array}{c}\text { Soma dos } \\
\text { quadrados }\end{array}$ & 528.693 & 766.833 & 498.55 & 610.223 & 244.206 \\
\hline Perfil & $\begin{array}{l}\text { Baixa mortalidade } \\
\text { materna e por } \\
\text { afecções originadas } \\
\text { do período perinatal }\end{array}$ & $\begin{array}{l}\text { Baixa mortalidade } \\
\text { por doenças } \\
\text { infecciosas e } \\
\text { parasitárias }\end{array}$ & \begin{tabular}{|} 
Elevada mortalidade \\
por causas \\
relacionadas à \\
Gravidez, parto e \\
puerpério. \\
\end{tabular} & $\begin{array}{c}\text { Elevada mortalidade } \\
\text { por afecções } \\
\text { originadas do } \\
\text { período perinatal }\end{array}$ & $\begin{array}{c}\text { Elevada mortalidade } \\
\text { por doenças } \\
\text { infecciosas e } \\
\text { parasitárias }\end{array}$ \\
\hline \multicolumn{6}{|c|}{ Grupo 3 - Doenças em Tecidos, Órgãos e Sistemas } \\
\hline Capítulo III & 0,52 & \begin{tabular}{|c|}
0,48 \\
\end{tabular} & \begin{tabular}{|c|}
0,50 \\
\end{tabular} & 1,51 & 0,72 \\
\hline Capítulo IV & 9,45 & 7,54 & 6,98 & 8,82 & 8,72 \\
\hline Capítulo XI & 5,68 & 5,33 & 3,80 & 4,56 & 4,43 \\
\hline Capítulo XII & 0,31 & 0,68 & 0,23 & 0,25 & 0,46 \\
\hline Capítulo XIII & 0,22 & 0,52 & 0,26 & 0,16 & 1,86 \\
\hline Capítulo XIV & 1,75 & 2,72 & 1,71 & 1,80 & 2,18 \\
\hline $\begin{array}{c}\text { Soma dos } \\
\text { quadrados }\end{array}$ & 1725.53 & 2782.73 & 1966.99 & 1243.4 & 288.367 \\
\hline Perfil & $\begin{array}{c}\text { Elevada mortalidade } \\
\text { por doenças } \\
\text { endócrinas } \\
\text { nutricionais e } \\
\text { metabólicas e } \\
\text { Doenças do aparelho } \\
\text { digestivo } \\
\end{array}$ & $\begin{array}{l}\text { Elevada mortalidade } \\
\text { por Doenças da } \\
\text { pele e tecido } \\
\text { subcutâneo, e } \\
\text { Doenças do aparelho } \\
\text { geniturinário }\end{array}$ & $\begin{array}{l}\text { Baixas taxas de } \\
\text { mortalidade }\end{array}$ & $\begin{array}{l}\text { Elevada mortalidade } \\
\text { por Doenças do } \\
\text { sangue, órgãos } \\
\text { hematopoiéticos } \\
\text { e transtornos } \\
\text { imunitários, }\end{array}$ & $\begin{array}{l}\text { Elevada mortalidade } \\
\text { por Doenças } \\
\text { do sistema } \\
\text { osteomuscular e do } \\
\text { tecido conjuntivo }\end{array}$ \\
\hline \multicolumn{6}{|c|}{ Grupo 4 - Transtornos Mentais e Sistema Nervoso } \\
\hline Capítulo V & 1,20 & \begin{tabular}{|l|}
2,85 \\
\end{tabular} & \begin{tabular}{|c|}
0,62 \\
\end{tabular} & 1,65 & 0,52 \\
\hline Capítulo VI & 3,02 & 2,04 & 1,67 & 1,06 & 0,66 \\
\hline $\begin{array}{c}\text { Soma dos } \\
\text { quadrados }\end{array}$ & 597.482 & 193.179 & 183.427 & 142.19 & 111.824 \\
\hline Perfil & $\begin{array}{l}\text { Elevada mortalidade } \\
\text { por Doenças do } \\
\text { Sistema nervoso }\end{array}$ & $\begin{array}{c}\text { Elevada mortalidade } \\
\text { por Transtornos } \\
\text { mentais e } \\
\text { comportamentais }\end{array}$ & $\begin{array}{l}\text { Baixa mortalidade } \\
\text { Transtornos mentais }\end{array}$ & $\begin{array}{c}\text { Mediana } \\
\text { mortalidade } \\
\text { Transtornos mentais } \\
\text { e comportamentais e } \\
\text { doenças do Sistema } \\
\text { nervoso } \\
\end{array}$ & $\begin{array}{c}\text { Baixa mortalidade } \\
\text { Transtornos mentais } \\
\text { e comportamentais e } \\
\text { doenças do Sistema } \\
\text { nervoso }\end{array}$ \\
\hline
\end{tabular}

Fonte: Sistema de Informação sobre Mortalidade (2020)

Com base nos resultados encontrados, foi possível observar que o perfil de transição epidemiológica vivenciado no Nordeste é caracterizado pela polarização epidemiológica, onde em algumas áreas predomina a mortalidade pelas DANT, e outras áreas com elevada carga de mortalidade infantil, materna e por doenças infectoparasitárias.

Os resultados do presente estudo revelam um perfil hegemônico de acometimento por doenças e agravos não transmissíveis, tendência em todo o mundo (CALAZANS; QUEIROZ, 2020; JAGANNATHAN et al., 2019; SCHLÜTERA et al., 2020). Diante desse cenário, a ascensão das doenças e agravos crônico-degenerativos se dá por intermédio de mudanças nas dinâmicas sociais, tais como envelhecimento populacional, número médio de anos vividos na idade adulta, além de condições socioeconômicas e estilo de vida (CALAZANS; QUEIROZ, 2020).

O Plano de Ações Estratégicas para o Enfrentamento das Doenças Crônicas Não Transmissíveis no Brasil (2011-2022) tem como meta reduzir a taxa de mortalidade em $2 \%$ ao ano até $2022 \mathrm{em}$ todos os estados (BRASIL, 2013). Apesar dos resultados do presente estudo demostrarem a elevada carga de mortalidade relacionada às DCNT na região nordeste, 
Confortin e colaboradores (2019) demonstraram que os estados da Bahia, Pernambuco e Sergipe reduziram a taxa de mortalidade em 2\% ao ano entre 2011 e 2014 . Por outro lado, Maranhão e Piauí apresentaram aumento de 1,7\% e 1\% ao ano, no mesmo período, nas taxas de mortalidade por doenças do aparelho circulatório, câncer, diabetes e doenças respiratórias crônicas (CONFORTIN et al., 2019).

A elevada carga de DANT na população brasileira está relacionada à exposição a fatores de risco como a alimentação inadequada, baixa atividade física, consumo de álcool e tabagismo. Além disso, quanto à complexidade do tratamento clínico, observa-se a limitada disponibilidade deste, restrito aos grandes centros urbanos (MANSUR; FAVARATO, 2016). Resultados da Pesquisa Nacional de Saúde de 2013 informam que $42,2 \%$ da população de 18 anos ou mais de idade da região nordeste relata ter pelo menos uma doença crônica não transmissível. A Região com maior prevalência de indivíduos com DCNT foi a Sul, com 52,1\%, seguida pelas Regiões Sudeste (46,1\%), Centro-Oeste (43,9\%), Nordeste (42,2\%) e Norte (37,2\%) (MALTA et al., 2017).

Em relação ao câncer, estudo desenvolvido por Barbosa et al. (2015) apontou tendências de aumento nas taxas de mortalidade por câncer em ambos os sexos até o ano de 2030, para as regiões Norte e Nordeste, enquanto que para as demais regiões brasileiras tendências decrescentes nessas taxas foram previstas. O estudo também concluiu que as acentuadas diferenças regionais e por sexo existentes na mortalidade por câncer no Brasil continuarão a aumentar até o ano de 2030. Para as próximas décadas, as projeções apontaram que a Região Nordeste apresentará a mais alta mortalidade por câncer do Brasil.

Em relação aos serviços de Atenção primária à saúde, o Nordeste registra ainda a maior cobertura de serviços de saúde pelo Sistema Único de Saúde (Paraíba e Piauí > 80\%), tendo cadastrada nas unidades de saúde da família a maioria da população. Considerando que a atenção primária à saúde pode reduzir consideravelmente essas doenças as na população, a alta cobertura registrada nestas regiões não tem se refletido em políticas de enfrentamento dessas doenças (STOPA et al., 2017; MALTA et al., 2013). Portanto, cabem às instituições de saúde intensificar políticas públicas de promoção e prevenção, capacitar profissionais para uma assistência qualificada, além de potencializar iniciativas de vigilância e busca ativa da população de risco, com o objetivo de restituir a tendência de queda da mortalidade por esses agravos nos estados com maior proporção de mortalidade por estas causas.

Por outro lado, evidencia-se forte concentração dos investimentos públicos nos estados da Bahia, Ceará e Pernambuco, o que pode explicar o alcance dessas metas nesses estados (TEIXEIRA et al., 2018). Além disso, as intervenções para a prestação de cuidados e promoção da saúde no SUS podem estar impulsionando o alcance dos objetivos em alguns estados a partir da expansão dos cuidados primários, a melhoria na prestação dos serviços de saúde e a distribuição de medicamentos para a população em risco (RIBEIRO et al., 2016).

$\mathrm{Na}$ análise dos clusters formados pela técnica de Skater, as causas externas são a principal carga de mortalidade proporcional entre os estados de Sergipe e Alagoas. Dentre as causas externas os acidentes e violência vêm ganhando destaque, onde dados relevam que, a cada ano, essas causas são responsáveis por mais de 5 milhões de mortes no mundo, sendo os jovens, negros e pobres os mais atingidos (SILVA et al., 2018). Evidencia-se ainda que, até 2033, as causas externas de mortalidade ainda devam aumentar em um número tão impressionante que terão efeito de arrefecimento na tendência do envelhecimento da população devido sua grande incidência na população jovem, como visto (SILVA; RAMALHO, 2015).

Dados do Instituto de Pesquisa Econômica Aplicada (IPEA) avaliou que nas regiões Norte e Nordeste situamse as sete UFs com maiores taxas de homicídios por 100 mil habitantes, sendo elas: Sergipe $(64,7)$, Alagoas $(54,2)$, Rio Grande do Norte $(53,4)$, Pará $(50,8)$, Amapá $(48,7)$, Pernambuco $(47,3)$ e Bahia $(46,9)$ (IPEA, 2018).

Rodriguez e Kovács (2005) argumentam que tal evento pode está relacionado diretamente ao fato da violência, antes praticada, especialmente, com o intuito de se adquirir bens materiais ter se banalizado, passando a funcionar como meio de expressão de indivíduos nas faixas etárias mais jovens. Santos (2007) reforça que, a falta de estrutura familiar, de políticas públicas específicas para os jovens, além da falta de emprego e, majoritariamente, o tráfico de drogas estejam entre os fatores que potencializam o risco de homicídios entre indivíduos desse grupo etário.

A elevada proporção de mortalidade por causas externas nesses estados também estão relacionados às elevadas taxas de mortalidade por suicídio. Santos e Barbosa (2017) analisaram a mortalidade por esta causa nos municípios nordestinos e destacaram que o estado de Sergipe apresentou taxas elevadas de mortalidade por suicídio (5,60 óbitos/100 mil hab.), estando atrás somente do estado de Piauí, Ceará e Rio Grande do Norte $(7,77 ; 6,92 ; 6,59$ óbitos/100 mil hab., respectivamente). Esses achados podem estar relacionados a fatores como desigualdade de renda, a baixa escolaridade, status de poder diminuído na vida social e familiar, taxa de desemprego, entre outros, podem ter impacto direto no comportamento suicida por meio de aspectos psicológicos como a depressão, o estresse, e a ansiedade (FERREIRA, 2015; FELIX et al., 2016; VIDAL; GONTIJO, 2013).

Outro agrupamento que foi identificado no nosso estudo está relacionado aos estados do Maranhão e Piauí, que correspondem às áreas em que prevalecem ainda a mortalidade infantil, materna e perinatal e as doenças infectoparasitárias, características inerentes às primeiras fases da transição epidemiológica e que destacam o processo de desigualdade no desenvolvimento territorial (ARAÚJO, 2012). Esse quadro ainda é observado nesses estados nordestinos apesar 
da redução significativa dos casos ao longo do tempo em decorrência da consolidação do SUS, do combate das doenças passíveis de imunizações e antibióticos, a ampliação do acesso aos serviços de saúde e a adoção de medidas de controle específicas (TEIXEIRA et al., 2018; DUARTE; BARRETO, 2012).

Segundo dados do IBGE (2010), o Piauí é considerado historicamente como um dos estados mais pobre do Brasil, possuindo renda per capita baixa (R\$ 408,27 reais), superior apenas a do estado do Maranhão, com $60,84 \%$ da população vivendo em baixa (pessoas ocupadas com rendimento até dois salários mínimos). Dessa forma, o perfil epidemiológico encontrado nesse estudo pode estar relacionado à própria construção regional do país, colocadas em evidência por Josué de Castro ainda em 1946, após traçar um perfil epidemiológico e dividir o país em áreas em que havia subnutrição ou fome, esta por sua vez de forma epidêmica ou endêmica (Castro, 1984). O perfil de mortalidade também pode possuir relação direta com o Índice de Desenvolvimento Humano (IDH), ou seja, a pobreza está intimamente associada à prevalência dessas doenças, causando profundo impacto econômico, social e político na região Nordeste (LINDOSO; LINDOSO, 2009).

Ainda que haja elevada mortalidade infantil nos estados do nordeste do Brasil, é importante ressaltar o êxito brasileiro quanto à meta 4 dos Objetivos de Desenvolvimento do Milênio em relação a mortalidade na infância. Em 2011, o país alcançou antecipadamente a meta ao atingir a taxa de 15,3 óbitos por mil nascidos vivos (NV) (VELOSO et al., 2019). Esses resultados foram decorrentes da inquestionável diminuição da pobreza observada nos últimos anos, além da ampliação da Estratégia de Saúde da Família (ESF) e consequentemente o fortalecimento do planejamento familiar e pré-natal, incentivo ao aleitamento materno, aumento da cobertura de imunização, cuidados as doenças prevalentes na infância, atenção ao parto, pós-parto e ao recém-nascido (FRIAS et al., 2013). Todavia, esses contrastes ainda persistem e tornam os indicadores de mortalidade bastante heterogêneos em todo o país (MENEZES et al., 2019). Em 2010, a região norte do país apresentou taxas que variavam de 11,0 a 21,8 óbitos/1.000 NV; na região nordeste, a TMI situou-se entre 11,9 e 17,8 óbitos/1.000 NV; nas regiões sudeste e sul as TMI variaram menos, situando-se entre 9,1 e 13,2 óbitos/1.000 NV. Por sua vez, as capitais da região centro-oeste apresentam TMI muito similares entre si, variando de 12,6 a 13,2 óbitos/1.000 NV (LIMA et al., 2017).

Desde o período da redemocratização no país, discute-se a importância da organização do sistema de saúde levando em consideração não só as unidades federativas, mas sim as desigualdades regionais, o comportamento dos agravos e as doenças em um território, muitas vezes havendo uma relação geográfica e viabilizando melhores resultados em detrimento de menores custos para os gestores locais (QUEIROZ et al., 2018).

A compreensão da regionalização dos agravos e doenças viabiliza, portanto, uma governança territorial, que diz respeito à coordenação de políticas e ações partilhadas, as quais se desenvolvem através de estratégias de desenvolvimento espaciais, processos de participação e identidade territorial (RIBEIRO; TANAKA; DENIS, 2017). Para a superação desses desafios, deve ser adotada uma abordagem multi e trans-escalar, para superar o pensamento hermético das regiões e considerar os diversos eventos que a perpassam (VIANA; IOZZI, 2019). As contribuições deste estudo, por sua vez, referem-se aos subsídios gerados para políticas públicas com ênfase nas ações preventivas, territorialmente determinadas, que poderão ser usadas como critério para repartição mais equânime dos recursos públicos, priorizando as regiões que apresentam os piores indicadores.

As análises aqui apresentadas orientam o olhar sob duas dimensões: regional e local. Ambas possuem desafios relacionadas à macro e micro política, que por sua vez contribuem para a compreensão do modelo polarizado de transição epidemiológica vivenciado na região nordeste. A principal limitação desse estudo diz respeito à qualidade da informação sobre mortalidade, passível de subregistro e sub-informação. Sobre o registro de óbitos no Brasil, deve-se mencionar que no passado houve problemas com a confiabilidade dos dados, especialmente nas regiões Norte e Nordeste, porém, o ganho de qualidade e os avanços no sistema de informações sobre mortalidade vêm obtendo notoriedade desde o ano 2000.

\section{Conclusão}

No presente estudo, é possível observar um panorama de polarização epidemiológica na região Nordeste. Os agravos não-transmissíveis: doenças do aparelho circulatório, neoplasias e causas externas tem se elevado rapidamente configurando um desafio para a saúde, pois detêm maior necessidade de atenção por parte das políticas públicas. Contudo, destacam-se os estados do Maranhão e Piauí onde ainda prevalecem às doenças infectocontagiosas e problemas relacionados ao período perinatal.

O comportamento espacial das doenças, as complexidades geográficas, cultural, as disparidades nas condições socioeconômicas de vida reforçam a necessidade de produzir informações que colaborem para formulação de estratégias de redução da morbimortalidade da população. Entretanto, por tratar-se de uma abordagem ecológica, o estudo apresenta limitações inerentes à sua metodologia, com relação à utilização das informações obtidas em bancos de dados secundários, estando sujeito ao viés da subnotificação. Apesar dessas limitações, esse estudo é relevante para o levantamento de áreas prioritárias para o desenvolvimento de ações de prevenção dos agravos em saúde.

\section{Referências}

ALI, M.K. et al. Noncommunicable diseases: three decades of global data show a mixture of increases and decreases in mortality 
rates. Health Affairs, v.34, n.9, p.1444-1455, 2015. doi: 10.1377/ hlthaff.2015.0570

ANDRADE, D.J.; SOUZA, A.A.M.; GOMES, C. Análise espacial por regionalização de áreas: o uso do método Skater no estudo da região metropolitana do vale do Paraíba e litoral Norte. Geografia, v. 40, n. 1, p. 53-71, 2015.

ARAÚJO, J. D. DE. Polarização epidemiológica no Brasil. Epidemiol. Serv. Saúde, v.21, n.4, p.533-538, 2012. doi: 10.5123/ S1679-49742012000400002

CALAZANS, J. A.; QUEIROZ, B. L. The adult mortality profile by cause of death in 10 Latin American countries (2000-2016). Rev. Panam. Salud Publica, v.44, p.1-9, 2020. doi: 10.26633/ RPSP.2020.1

CONFORTIN, S.C et al. Mortalidade prematura pelas principais doenças crônicas não transmissíveis nos estados do Brasil. Rev. Bras. Enferm., v. 72, n. 6, p. 1588-1594; 2019. doi: 10.1590/00347167-2018-0701

DIAS FILHO, J.M.; CORRAR, L. J.; PAULO, E. Análise multivariada. São Paulo: Atlas, 2007.

DUARTE, E. C.; BARRETO S. M. Transição demográfica e epidemiológica: a Epidemiologia e Serviços de Saúde revisita e atualiza o tema. Epidemiol. Serv. Saúde; v.21, n. 4, p.529-532, 2012. doi: 10.5123/S1679-49742012000400001

FÉLIX, T.A et al. Fatores de risco para tentativa de suicídio: produção de conhecimento no Brasil. Revista Contexto \& Saúde, v. 16, n. 31, p. 173-185, 2016. doi: 10.21527/21767114.2016.31.173-185

FERREIRA, A.J. The suicidal behavior in Brazil and in the world. Rev. Bras. Psicologia. v.2, n.1, p.15-28, 2015.

FRIAS, P. G. et al. Correcting vital information: estimating infant mortality, Brazil, 2000-2009. Rev. Saúde Pública, v.47, n.6, p.1048-1058; 2013. doi: 10.1590/S0034-8910.2013047004839

INPE. Análise Espacial de Dados Geográficos, Curso de PósGraduação, São José dos Campos: INPE, 2006.

Instituto de Pesquisa Econômica Aplicada (IPEA). Atlas da Violência 2018. Brasília: IPEA, 2018. Disponível em:< http://www. ipea.gov.br/portal/index.php?option $=$ com content $\&$ view $=$ article \&id $=33410 \&$ Itemid $=432>$.

JAGANNATHAN R. et al. Global Updates on Cardiovascular Disease Mortality Trends and Attribution of Traditional Risk Factors. Current Diabetes Reports, v. 44, n. 19, p. 1-12, 2019. doi: 10.1007/s11892-019-1161-2

KABUDULA, C. W. et al. Progression of the epidemiological transition in a rural South African setting: findings from population surveillance in Agincourt, 1993-2013. BMC Public Health, v. 17, n. 1, p. 424, 2017. doi: 10.1186/s12889-017-4312-x

LIMA, J.C et al. Population-based study on infant mortality. Ciênc. Saúde Coletiva, v.22, p.931-939, 2017. doi: 10.1590/1413-81232017223.12742016

LINDOSO, J.A.L.; LINDOSO, A.A.B.P. Neglected tropical diseases in Brasil. Rev. Inst. Med. Trop. São Paulo. v.51, n.5, p.247-253, 2009. doi: 10.1590/s0036-46652009000500003

MALTA, D.C et al. Doenças crônicas não transmissíveis e a utilização de serviços de saúde: análise da Pesquisa Nacional de Saúde no Brasil. Rev. Saúde Pública, v.51, p.4s, 2017. doi: 10.1590/s1518-8787.2017051000090

MALTA, Deborah Carvalho et al. Family health strategy coverage in Brazil, according to the National Health Survey, 2013. Ciênc. Saúde Coletiva, v.21, p.327-338, 2016. doi: 10.1590/141381232015212.23602015
MANSUR, A.P.; FAVARATO, D. Tendências da taxa de mortalidade por doenças cardiovasculares no Brasil, 19802012. Arq. Bras. Cardiol., v.107, n.1, p.20-25, 2016. doi: 10.5935/ abc. 20160077

MARINHO, F.; PASSOS, V.M.A.; FRANÇA, E. B. Novo século, novos desafios: mudança no perfil da carga de doença no Brasil de 1990 a 2010. Epidemiol. Serv. Saúde, v.25, n.4, p.713-724, 2016. doi: 10.5123/s1679-49742016000400005

MENDOZA, W.; MIRANDA, J. J. Global Shifts in Cardiovascular Disease, the Epidemiologic Transition, and Other Contributing Factors. Cardiol. Clin., v.35, n.1, p.1-12, 2017. doi: 10.1016/j. ccl.2016.08.004

BRASIL. Ministério da Saúde. Plano de Ações Estratégicas para o Enfrentamento das Doenças Crônicas Não Transmissiveis (DCNT) no Brasil (2011-2022); Brasília: Ministério da Saúde, 2013

QUEIROZ, R. DE et al. Princípios para a gestão da clínica: conectando gestão, atenção à saúde e educação na saúde. Ciênc. Saúde Coletiva, v.23, n.12, p.4249-4257, 2018. doi: $10.1590 / 1413-812320182312.32262016$

RIBEIRO, A. L. et al. Cardiovascular health in Brazil: trends and perspectives. Circulation, v. 133, n. 4, p. 422-33, 2016. doi: 10.1161/CIRCULATIONAHA.114.00872

RIBEIRO, C.C.P.; ABRANTES, L.A.; SILVA, J.V. Desenvolvimento municipal: uma análise multivariada para os municípios mineiros. In: CONGRESSO BRASILEIRO DE CUSTOS-ABC. 2014.

RIBEIRO, P. T.; TANAKA, O. Y.; DENIS, J.-L. Governança regional no Sistema Único de Saúde: um ensaio conceitual. Ciênc. Saúde Coletiva, v.22, n.4, p.1075-1084, 2017. doi: 10.1590/1413-81232017224.28102016

RODRIGUES, N.C.P et al. Temporal and spatial evolution of maternal and neonatal mortality rates in Brazil, 19972012. J. Pediatr., v.92, n.6, p.567-573, 2016. doi: 10.1016/j. jped.2016.03.004

RODRIGUEZ, C. F.; KOVÁCS, M. J. O que os jovens têm a dizer sobre as altas taxas de mortalidade na adolescência? Imaginário. v. 1, n.11, p. 111-136, 2005.

SANTOS, E.G.O.; BARBOSA, I.R. Conglomerados espaciais da mortalidade por suicídio no nordeste do Brasil e sua relação com indicadores socioeconômicos. Cad. Saúde Coletiva, v.25, n.3, p.371-378, 2017. doi: 10.1590/1414-462x201700030015

SANTOS, J. E. F. Homicídio entre Jovens de uma Periferia de Salvador, Bahia: um relato de experiência sobre a violência e o desenvolvimento humano. Rev. Bras. Crescimento Desenvolv. Hum., v.17, n.3, p.72-83, 2007.

SCHLÜTERA, B.-S. et al. Long-term trends in seasonality of mortality in urban Madagascar: the role of the epidemiological transition. Global Health Action, v.13, p.1-13, 2020. doi: 10.1080/16549716.2020.1717411

SCHRAMM, J.A.A et al. Transição epidemiológica e o estudo de carga de doença no Brasil. Ciênc. Saúde Coletiva, v.9, p. 897908, 2004. doi: 10.1590/S1413-81232004000400011

SILVA, J.R.; RAMALHO, W. M. Cenário epidemiológico do Brasil em 2033: uma prospecção sobre as próximas décadas. Fundação Oswaldo Cruz: Rio de janeiro, 2015.

SILVA, R. A. et al. Mortalidade por causas externas em jovens no estado da Bahia. Rev Fund Care, v. 10, n. 12, p. 46-51, 2018. doi: $10.1590 / \mathrm{S} 0102-311 \mathrm{X} 2000000400024$

STOPA, S.R et al. Acesso e uso de serviços de saúde pela população brasileira, Pesquisa Nacional de Saúde 2013. Rev Saúde Pública, 
v.51, p.3s, 2017. doi: 10.1590/s1518-8787.2017051000074

TEIXEIRA, M. G. et al. Conquistas do SUS no enfrentamento das doenças transmissíveis. Ciênc. Saúde Coletiva, v. 26, n. 6, p. 1819-1828, 2018. doi: 10.1590/1413-81232018236.08402018

VASCONCELOS, C.Hetal. Fatores ambientais e socioeconômicos relacionados à distribuição de casos de leptospirose no Estado de Pernambuco, Brasil, 2001-2009. Cad. Saude Colet., v. 20, n. 1, p. 49-56, 2012.
VELOSO, F.C.S et al. Analysis of neonatal mortality risk factors in Brazil: a systematic review and meta-analysis of observational studies. J. Pediatr., v.95, n.5, p.519-530, 2019. doi: 10.1016/j. jped.2018.12.014

VIANA, A. L. D’ÁVILA; IOZZI, F. L. Enfrentando desigualdades na saúde: impasses e dilemas do processo de regionalização no Brasil. Cad. Saúde Pública, v.35, n.2, p 1-12, 2019. doi: 10.1590/0102-311x00022519. 